\title{
Diffusion-weighted ASPECTS as a functional outcome predictor in patients with acute ischemic stroke treated with endovascular therapy up to 24 hours
}

C. Ciardi, J. Cirio, M.D. Buezas, J. Vila, M. Caballero, M. Lopez, J.P Chudyk Huberuk, G. Maida, P. Lylyk.

\author{
Neurology Department, Clínica La Sagrada Familia-ENERI \\ Buenos Aires, Argentina
}

\section{BACKGROUND}

In acute ischemic stroke patients Diffusion-weighted ASPECTS is correlated with infarct volume and also is an independent factor of functional outcome.

\section{OBJECTIVES}

Our aim is to assess the impact of pre-treatment

Diffusion-weighted ASPECTS on outcomes after endovascular treatment (EVT) within 24 hs.

\section{MATERIALS AND METHODS}

Patients aged I 8-80 years ago with acute ischemic stroke with large vessel occlusion from anterior circulation eligible for EVT within 24 hours were included in this descriptive, monocentric, ambispective study.

Vascular neurologists and neurorradiologist measured ASPECTS on DWI according to the method by Barber et al. Diffusionweighted

ASPECTS were grouped in $\geq 6$ (Group A) or $<6$ (Group B) The primary outcome measured was a modified Rankin scale $(\mathrm{mRs}) \leq 2$ at 90 days. Secondary outcomes included a

successful reperfusion, defined as a modified Thrombolysis in Cerebral Infarction $(\mathrm{mTICl})$ scale $2 \mathrm{~b} / 3$, mortality rate at 90 days and symptomatic intracranial hemorrhage $(\mathrm{SIH})$

Descriptive and frequency statistical analyses were obtained using SPSS 21 software. Categorical variables are presented as absolute values and percentages, and the continuous variables are presented as median \pm SD if normally distributed or median (interquartile intervals) if not normally distributed. Statistical significance for intergroup differences was assessed by Pearson $\mathrm{X} 2$ or Fisher exact test for categorical variables and by Student $t$ or Mann-Whitney $U$ test for continuous variables.

\section{RESULTS}

We included 83 patients, 56 in Group A and 27 in Group B. Demographics data, risk factors, $\mathrm{SIH}$ and successful reperfusion did not show significant differences.

The rate of $\mathrm{mRS} \leq 2$ at 90 days was 69,6\% in Group $A$ as compared with $29.6 \%$ in Group B ( $P=0.0006 \mathrm{OR}=5.4 \mathrm{IC}=1.9$ -

14.8), the rate of mortality was 3,6\% Group A while in Group B was $19 \%(P=0,022 \mathrm{OR}=0.16 \mathrm{IC}=0.02-0.90)$

\section{CONCLUSION}

Diffusion-weighted ASPECTS $\geq 6$ seems to be a good parameter predicting favorable outcome among patients treated by EVT within 24 hs.

\begin{tabular}{|l|c|c|c|}
\hline Baseline Characteristics and Metrics & Group A (ASPECTS $\geq$ 6) & Group B (ASPECTS < 6) & P \\
\hline Age-yr- Mean-SD & $64(+/-11)$ & $60(+/-13)$ & 0.20 \\
\hline Male sex-no. (\%) & $30(54 \%)$ & $13(48 \%)$ & 0.64 \\
\hline Atrial Fibrillation -no. (\%) & $7(13 \%)$ & $7(26 \%)$ & 0.12 \\
\hline Diabetes mellitus -no. (\%) & $8(14 \%)$ & $4(15 \%)$ & 0.94 \\
\hline Hypertension -no. (\%) & $36(64 \%)$ & $18(67 \%)$ & 0.83 \\
\hline NIHSS admission -Median-IQR & $10(6-14)$ & $17(15-19)$ & 0.0001 \\
\hline Core Volumen DWI -Median-IQR & $12(4-29)$ & $72(54-127)$ & 0.0001 \\
\hline $\begin{array}{l}\text { Time from stroke onset to admission } \\
\text { Min-Median (IQR) }\end{array}$ & $205(85-350)$ & $255(90-485)$ & 0.62 \\
\hline $\begin{array}{l}\text { Time from stroke onset to groin puncture } \\
\text { Min-Median (IQR) }\end{array}$ & $305(200-445)$ & $342(189-554)$ & 0.54 \\
\hline $\begin{array}{l}\text { Time from stroke onset to revascularization } \\
\text { Min-Median (IQR) }\end{array}$ & $351(244-469)$ & $394(216-573)$ & 0.68 \\
\hline $\begin{array}{l}\text { Time from door to revascularization } \\
\text { Min-Median (IQR) }\end{array}$ & $114(81-154)$ & $117(82-152)$ & 0.78 \\
\hline
\end{tabular}

Primary outcomes. mRS at 90 days.

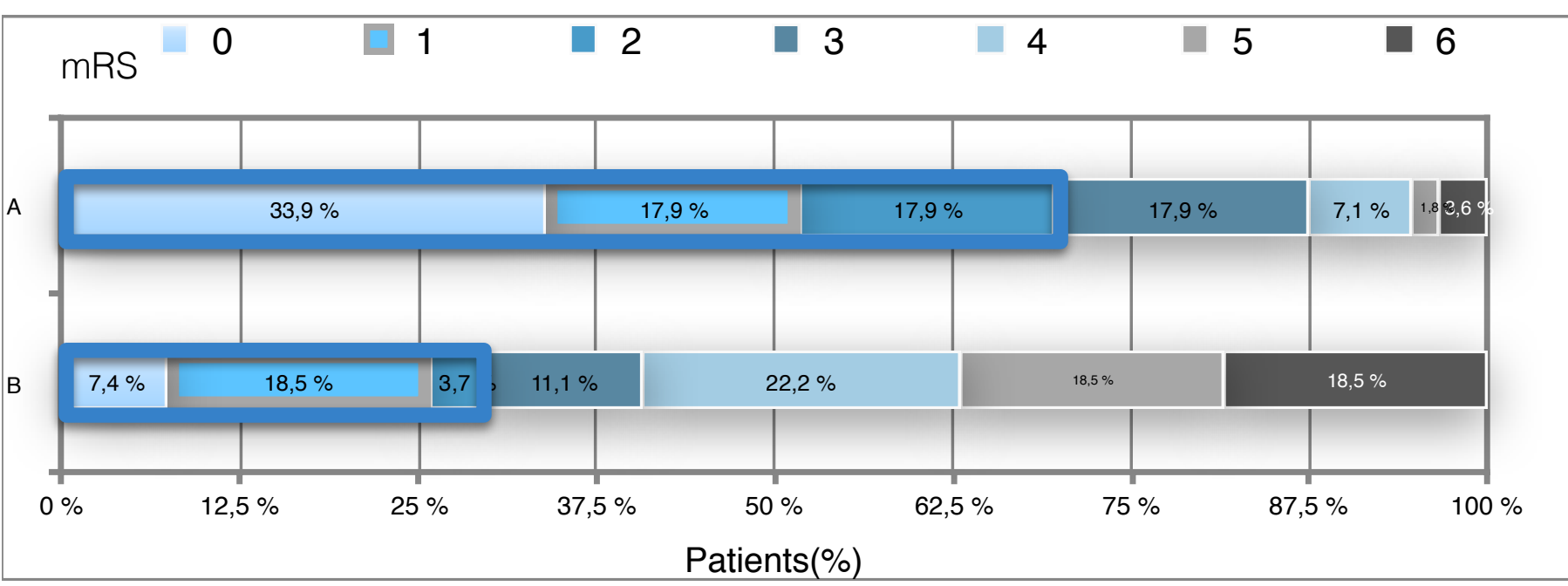

Secondary outcomes. mTICI
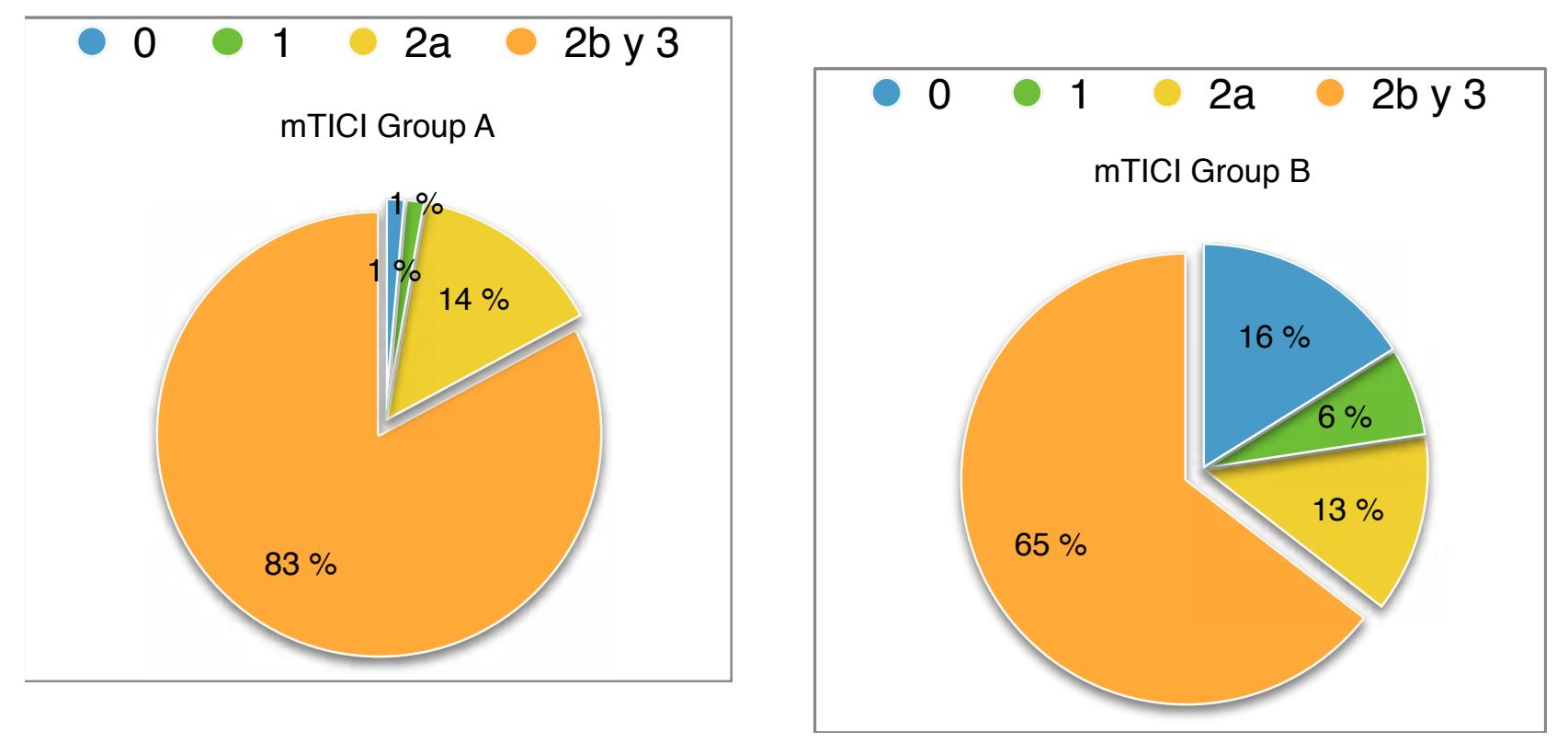

Safety outcome.

Symptomatic intracranial hemorrhage.

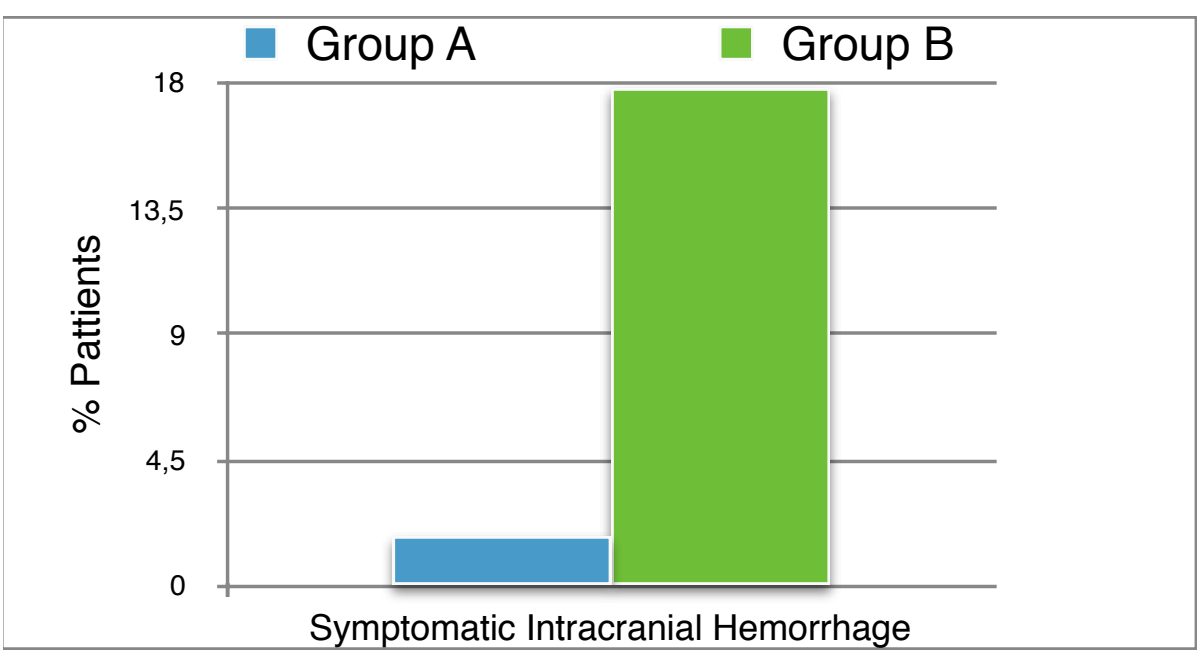

